\section{Feste Kombination von Budesonid und Formoterol zur Therapie des Asthma bronchiale}

\section{Rationale einer Kombinationstherapie}

Asthma bronchiale wird bis auf die nur intermittierend ausgeprägte Erkrankung (Stufe I) mittels verschiedener Pharmaka kombiniert therapiert, wobei sowohl bronchodilatatorische als auch antiinflammatorisch wirkende Substanzen gleichzeitig zur Anwendung kommen. Es liegt daher sowohl aus Gründen der Praktikabilität als auch der Effektivität nahe in der Wirkung sich ergänzende und in gleicher Weise applizierbare Substanzen trotz unterschiedlicher Wirkungsmechanismen zu kombinieren.

An Kombinationspräparate sind eine Reihe von Anforderungen zu stellen [1]:

1. Jede Einzelkomponente der Kombination trägt zu der beabsichtigten Wirkung bei.

2. Die Komponenten passen hinsichtlich Wirkungseintritt, Wirkungsdauer, Dosierungsintervall und Applikationsform zueinander.

3. Die Komponenten liegen in einem Mengenverhältnis vor, welches das Präparat wirksam und unbedenklich für eine ausreichend große Zahl von Patienten macht, die der gleichzeitigen Behandlung mit beiden Einzelkomponenten bedürfen.

Feste Kombinationen inhalierbarer Glukokortikosteroide und langwirkender $\beta_{2}$-Sympathomimetika können diese Anforderungen erfüllen.

Im Folgenden soll ein neues inhalatives Kombinationspräparat, das Budesonid und Formoterol enthält, diskutiert werden, das in einem Trockenpulverinhalationssystem (Turbohaler) 2001 in Deutschland verfügbar sein wird.

\section{Klinische Effektivität einer festen Kombinationstherapie in der Asthma-Therapie}

Trotz der Empfehlung in fast allen Leitlinien zur AsthmaTherapie, mittelschwer- und schwerkranke Asthmapatienten inhalativ mit einem langwirksamen $\beta_{2}$-Sympathomimetikum und einem Glukokortikosteroid zu behandeln, fehlte bis Mitte der 90er Jahre der Nachweis des additiven Effekts einer solchen kombinierten Anwendung. Inzwischen belegen jedoch mehrere klinische Prüfungen an einer großen Zahl von

Pneumologie 2001; 55: 159-162

(c) Georg Thieme Verlag Stuttgart · New York ISSN 0934-8387
A. Gillissen ${ }^{1}$, R. Buhl², H. Magnussen 3 , G. Menz ${ }^{4}$, K. Rabe ${ }^{5}$, C. Vogelmeier ${ }^{6}$, H. Worth ${ }^{7}$

${ }^{1}$ Städtisches Klinikum St. Georg, Robert-Koch-Klinik, Leipzig

2 Schwerpunkt Pneumologie, III. Medizinische Klinik und Poliklinik, Universitätsklinikum Mainz

${ }^{3}$ Krankenhaus Großhansdorf

${ }^{4}$ Hochgebirgsklinik Davos, Davos-Wolfgang, Schweiz

${ }^{5}$ Dept. of Pulmonology, Medical Center, Leiden University Medical Center, Niederlande

${ }^{6}$ Klinik für Innere Medizin, Schwerpunkt Pneumologie, Klinikum der Philipps-Universität, Marburg

${ }^{7}$ Medizinische Klinik I, Klinikum Fürth

Patienten mit klinisch sehr gut charakterisiertem Asthma, dass die Kombination eines langwirkenden $\beta_{2}$-Sympathomimetikums (Formoterol oder Salmeterol) mit einem inhalativen Glukokortikosteroid (Budesonid oder Fluticason) zu einer besseren Symptomkontrolle, einer Lungenfunktionsverbesserung und zu einer Reduktion der Exazerbationsrate führt. Die Kombination ist dabei effektiver, als eine Verdoppelung der inhalativen Glukokortikosteroiddosis [2-6].

Ein, bezogen auf die Lungenfunktion möglicher überadditiver Effekt einer solchen Kombination ist Gegenstand intensiver wissenschaftlicher Diskussion [7-9].

Die FACET-Studie (formoterol and corticosteroids establishing therapy) belegte, dass mittels Turbohaler appliziertes Formoterol zusätzlich zu einer niedrigen $(100 \mu \mathrm{g} / 2 \times 1 /$ Tag Budesonid) oder höheren Kortikosteroiddosis $(400 \mu \mathrm{g} / 2 \times 1 /$ Tag Budesonid) die Symptomkontrolle und die Lungenfunktion verbessern kann, und dass diese Effekte unverändert über 12 Behandlungsmonate nachweisbar waren [4]. Zudem scheint dieser Ansatz auch kosteneffektiv zu sein [10]. Auch bei Kindern erbrachte die kombinierte Gabe eines langwirksamen $\beta_{2}$-Sympathomimetikums mit einem inhalativen Glukokortikosteroid im Vergleich zu der Monotherapie mit den Einzelsubstanzen eine, wenn auch geringe zusätzliche Lungenfunktionsverbesserung $\left(\mathrm{FEV}_{1}\right.$ und Peak-Flow) und eine Abnahme der Asthmasymptome [11]. Unklar blieb, ob die bronchiale Entzündungsreaktion durch die Therapie mit Formoterol lediglich maskiert wurde. Im Vergleich der Budesonid-Gruppe $(2 \times 400 \mu \mathrm{g} / \mathrm{Tag})$ gegenüber der Budesonid- $(2 \times 100 \mu \mathrm{g})$ Formoterol (12 $\mu \mathrm{g} 2 \times 1 / \mathrm{Tag})$-Kombinationsgruppe zeigten sich jedoch keinerlei Unterschiede in der Sputum-Eosinophilenzahl und -ECP-Konzentration (eosinophilic cationic proteine), so dass eine durch das $\beta_{2}$-Mimetikum verursachte Maskierung ausgeschlossen werden konnte [12]. Ähnliche Ergebnisse ließen sich auch mit Fluticason und Salmeterol erzielen [7 -9].

Als Konsequenz wurden und werden aktuell Formulierungen entwickelt, die beide Wirkprinzipien durch die gleichzeitige Applikation verbinden. Dabei ist die feste Kombination in einem Inhalator nachweisbar in gleicher Weise effektiv wie die getrennte Gabe der Einzelsubstanzen kurz hintereinander [7,13-17]. Seit 1999 ist in Deutschland eine feste Kombination aus Salmeterol und Fluticason in drei verschiedenen Dosierungen (Salmeterol/Fluticason 50/100 $\mu \mathrm{g}, 50 / 250 \mu \mathrm{g}$ und $50 / 500 \mu \mathrm{g}$ ) verfügbar [14]. Eine Expertengruppe erachtete unter gewissen Voraussetzungen (Langzeittherapie, stabile Erkrankungssituation) den Einsatz dieses Präparates in der Asthmatherapie als sinnvoll [18]. 


\section{Feste Kombination aus Budesonid und Formoterol}

2001 wird in Deutschland ein zweites inhalatives Kombinationspräparat mit einem langwirksamen $\beta_{2}$-Rezeptoragonisten (Formoterol, $6 \mu \mathrm{g} / \mathrm{Hub}$ ) und einem Glukokortikosteroid (Budesonid, $200 \mu \mathrm{g} / \mathrm{Hub}$ ) in einem Trockenpulverinhalationssystem (Turbohaler) zur Therapie des Asthma bronchiale verfügbar sein. Eine Reihe von Studien, die auf dem Kongress der European Respiratory Society (ERS) 2000 vorgestellt wurden und bislang nur als Abstract vorliegen (s.u.), belegen die Wirksamkeit dieses Präparates.

\section{Klinische Wirkung bei Erwachsenen}

In der klinischen Wirksamkeit unterschieden sich bei Patienten ( $\mathrm{n}=362$; Therapiedauer: 12 Wochen) mit einem Asthma bronchiale die getrennte Applikation von Budesonid und Formoterol (Budesonid $200 \mu \mathrm{g}$ Formoterol $6 \mu \mathrm{g}$ beide $2 \times 2$ / Tag) nicht von der festen Kombination ( $200 \mu \mathrm{g} / 6 \mu \mathrm{g} 2 \times 2 / \mathrm{Tag})$. Beide waren aber erwartungsgemäß effektiver als die Budesonid Monotherapie (200 $\mu \mathrm{g} 2 \times 2 / \mathrm{Tag})$. Die $\mathrm{FEV}_{1}$, der SymptomScore und der Peak-Flow am Morgen und am Abend waren gegenüber der Budesonid Monotherapie signifikant $(p<0,001)$ verbessert. Signifikante Unterschiede zwischen den beiden Formoterol/Budesonid-Behandlungsgruppen (getrennte Einzelgaben Formoterol/Budesonid und feste Kombination) errechneten sich nicht. Allerdings wies die Gruppe mit der festen Kombination in der Tendenz (keine Signifikanz) einen initial schnelleren Wirkeintritt, einen verminderten Bedarf an Notfallmedikation und einen geringeren Symptomen-Score auf als die Patienten, die getrennt die Prüfsubstanzen erhielten [19]. Außerdem ließen sich mit beiden Kombinationen gegenüber einer Budesonid Monotherapie das Exazerbationsrisiko um ca. $40 \%$ senken $(p<0,01)$ und die Zahl der Tage, an denen das Asthma besser kontrolliert war (keine Symptome, keine Notfallmedikation, kein nächtliches Erwachen wegen Dyspnoe) signifikant $(p<0,001)$ erhöhen $[20,21]$

\section{Klinische Wirkung bei Kindern und Jugendlichen}

Auch bei Kindern und Jugendlichen mit einem Asthma bronchiale im Alter zwischen 4 und 17 Jahren konnte die klinische Wirksamkeit der festen Kombination mit Budesonid und Formoterol $(100 \mu \mathrm{g} / 6 \mu \mathrm{g} 2 \times 2 / \mathrm{Tag})$ belegt werden. Bei 81 Kindern und Jugendlichen ließ sich durch die fixe Kombination die 12-h Durchschnitts-FEV ${ }_{1}$ um $5 \%(p=0,04)$ gegenüber der Budesonid Monotherapie (100 $\mu \mathrm{g} 2 \times 2$ /Tag; Studiendauer 12 Wochen) steigern, was einem Nettogewinn von ca. $300 \mathrm{ml}$ oder $>14 \% \mathrm{FEV}_{1}$ entspricht [22]. Bestätigend dazu stieg auch der Peak-Flow (Morgen- und Abendwert) an. So ließ sich mit der festen Kombination ein Nettogewinn von 23 l/min, d.h. mehr als doppelt so viel als mit einer Budesonid Monotherapie (11 1/min) erreichen [23]. Bezüglich der Nebenwirkungen unterschieden sich die Gruppen feste Kombination vs. getrennte Gabe der Einzelsubstanzen vs. Budesonid Monotherapie nicht signifikant $[21,23]$.

\section{Compliance}

Es ist zu vermuten, dass die kombinierte Gabe eines langwirksamen $\beta_{2}$-Sympathomimetikums und eines Glukokortikosteorids in einer festen Kombination nicht nur $\mathrm{zu}$ einer Steigerung der Patienten-Compliance führt, sondern gleich- zeitig auch eine begleitende in der Praxis oft vernachlässigte Steroidapplikation zwangsläufig sicherstellt. Diese Frage ist allerdings in einer kontrollierten Studie bisher noch nicht untersucht worden [18]. Diese Vermutung basiert auf dem Nachweis, dass die Compliance bei einer häufigeren täglichen Anwendung eines Dosieraerosols von z.B. > 60\% $(2 \times 1 \mathrm{Hub} /$ Tag) auf $<20 \%$ sinkt (bei $4 \times 1$ Hub/Tag) [24]. Auch bei Erwachsenen bestätigte sich kürzlich eine in Abhängigkeit zur Einnahmehäufigkeit bestehende hohe Variationsbreite (40\%) der Patienten-Compliance [25]. Durch die niedrige Dosis beider Einzelsubstanzen lässt sich durch Mehrfachdosierung möglicherweise eine bedarfsadaptierte Dosisanpassung erzielen, da Arzt und Patient in Abhängigkeit von der Erkrankungsschwere die Dosis steigern oder auch wieder reduzieren können (Abb.1). Diese wichtige Frage ist Gegenstand einer aktuellen klinischen Prüfung [26].

Wie generell bei der Therapie chronischer Erkrankungen, ist es auch bei Asthma bronchiale eine häufige klinische Beobachtung, dass bei guter Symptomkontrolle die Compliance der Patienten abnimmt [27-31]. Dies gilt besonders für die Therapie mit inhalierbaren Kortikoiden (Kortison-Angst) [32 - 34]. Der subjektiv empfundene schnelle Wirkeintritt des Formoterols in der Kombination könnte zur Verbesserung der Langzeitcompliance beitragen [27,32,34].

Darüber hinaus müssen die Patienten nur noch mit einem statt mit zwei Inhalatoren zwei verschiedene Medikamente inhalieren. Es ist wahrscheinlich, dass diese Vereinfachung zu einer besseren Akzeptanz der Langzeittherapie und zu einer Steigerung der Compliance beiträgt [32,34]. Die Abnahme der Compliance mit steigender Anzahl verordneter Medikamente ist belegt [27-29,31].

\section{Verträglichkeit}

An 14 Asthmatikern wurde in einer randomisierten doppeltverblindeten in einem double-dummy-Design durchgeführten Untersuchung die Auswirkungen einer hohen Tagesdosis

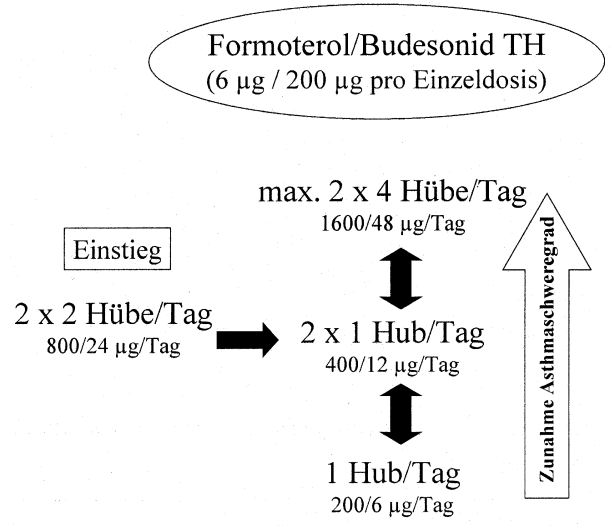

Abb. 1 Therapieoptionen mit der neuen festen Kombination Budesonid/Formoterol zur Therapie des Asthma bronchiale. Die niedrige Basisdosis von $6 \mu \mathrm{g}$ Formoterol $/ 200 \mu \mathrm{g}$ Budesonid könnte in Abhängigkeit von der Erkrankungsschwere bis auf max. $2 \times 24 \mu \mathrm{g} / 800 \mu \mathrm{g}$ $(=2 \times 4$ Hübe) pro Tag gesteigert werden (bezogen auf die max. Dosierung der Einzelkomponenten in der Dauertherapie). Allerdings gibt es noch keine Untersuchungen zur bedarfsorientierten Langzeittherapie. Dieser Aspekt wird in der ATACO-Studie untersucht [26]. 
der festen Budesonid/Formoterol-Kombination (Basismedikation: $400 \mu \mathrm{g}$ Budesonid/12 $\mu \mathrm{g}$ Formoterol und zusätzlich $2000 \mu \mathrm{g}$ Budesonid und $60 \mu \mathrm{g}$ Formoterol) geprüft. Die Veränderungen von Blutdruck, Serum-Kalium, Blutzucker, Plasmalaktat und QT-Intervallzeit entsprachen ungefähr denen, die mit der Formoterol Monotherapie $(60 \mu \mathrm{g}=10$-fache Dosis eines einzelnen Hubs) erzielt wurden. So stieg z.B. der Puls $4 \mathrm{~h}$ nach Applikation von ca. 60/min auf 80/min (Placebo: 74/ min) an und das Serum-Kalium fiel von ca. $4 \mathrm{mmol} / \mathrm{l}$ auf 3,6 mmol/l 2 - $4 \mathrm{~h}$ nach Applikation ab [35].

\section{Indikationen zum Einsatz einer festen Kombination eines langwirksamen $\boldsymbol{\beta}_{2}$-Sympathomimetikums und eines Glukokortikosteroids}

Entsprechend nationaler und internationaler Therapieempfehlungen wird erst das mittelschwere-persistierende Asthma mit einem inhalierbaren Kortikoid und einem langwirkenden $\beta_{2}$-Sympathomimetikum behandelt [36-39]. Mit den Einzelsubstanzen behandelte Patienten in einer stabilen Erkrankungsphase können unmittelbar auf das entsprechende Kombinationspräparat umgestellt werden. Bei der Ersteinstellung wurde bisher empfohlen, die Therapie individuell mit den Einzelkomponenten zu beginnen. Bei gutem Ansprechen auf die Therapie und stabilem Erkrankungsverlauf kann bei erneuter Verordnung auf die Kombination übergegangen werden. Möglicherweise könnte auf diese Vorgehensweise bei der neuen Formoterol/Budesonid-Formulierung verzichtet werden, da der $\beta_{2}$-Mimetikaanteil mit $6 \mu \mathrm{g}$ Formoterol genügend Spielraum für eine symptomorientierte Dosisanpassung erlaubt.

\section{Differenzielle Indikation des Einsatzes einer festen Kombination eines langwirksamen $\boldsymbol{\beta}_{2}$-Mimetikums und eines Kortikoids}

Bezüglich der Pharmakokinetik ist Formoterol gegenüber Salmeterol durch einen schnelleren Wirkungseintritt charakterisiert, der mit dem von Salbutamol vergleichbar ist [40]. Aus diesem Grund ist Formoterol-Turbohaler auch zur Bedarfstherapie des Asthma bronchiale zugelassen [41]. Unter Berücksichtigung der in den Studien verwendeten unterschiedlichen Applikationssystemen ist, bezogen auf die Wirkdauer und Wirkstärke von einer klinischen Äquipotenz üblicher Dosen von Formoterol und Salmeterol auszugehen [42]. Auch das Nebenwirkungsspektrum beider Substanzen ist ähnlich: Es ist in erster Linie mit Tremor und Tachykardien zu rechnen, den typischen unerwünschten Wirkungen aller $\beta_{2}$ Mimetika. Die Frequenz dieser Nebenwirkungen ist niedrig und unterscheidet sich zwischen einzelnen $\beta_{2}$-Mimetika nicht in klinisch relevanter Weise $[42,43]$.

Aufgrund der besonderen Pharmakokinetik von Formoterol bietet die feste Kombination aus Budesonid und Formoterol den Vorteil eines raschen Wirkungseintritts mit der Möglichkeit mit ein und demselben Inhalationssystem symptomadaptiert zu therapieren und erscheint besonders für Patienten geeignet:

a) die durch die unmittelbare Erfahrung einer bronchodilatatorischen Wirkung (und evtl. „gewohnter“ Nebenwirkungen) in der Therapietreue bestätigt werden,

b) die Probleme mit der Unterscheidung zwischen Bedarfsund Dauermedikation haben, da die feste Kombination mit
Budesonid und Formoterol auch im Notfall rasch wirksam ist,

c) die an einer instabilen Erkrankungsform leiden, da die morgendliche und abendliche Dosis vom behandelnden Arzt problemlos ohne Neuverordnung erhöht werden und nach einigen Tagen wieder verringert werden kann.

Sicher erscheint, dass die feste Kombination für die generelle Anwendung bei der COPD (chronic obstructive pulmonary disease) wegen der dort zu erwartenden hohen Steroid-NonResponderrate von 80-90\% nicht generell empfohlen werden kann [44].

\section{Resumee}

Zusammenfassend handelt es sich bei den neuen inhalativen Kombinationspräparaten um eine für die tägliche Arbeit in der Praxis hilfreiche und klinisch sinnvolle Ergänzung der Asthma-Therapie. Diese Aussage gilt zunächst nur für die Stufen III und IV. Allerdings bleiben zum gegenwärtigen Zeitpunkt noch viele Fragen offen, z.B. ob eine Anwendung, insbesondere des neuen Formoterol-haltigen Präparates schon in der Stufe II möglich und sinnvoll ist, ob auch eine einmalige Gabe bei leichteren Asthmaformen ausreichen könnte, und ob sich feste Kombinationen bereits für die Initialtherapie als geeignet erweisen.

\section{Literatur}

${ }^{1}$ Fülgraff G, Palm D. Pharmakotherapie - klinische Pharmakologie. Stuttgart: Gustav Fischer Verlag, 1995

${ }^{2}$ Greening AP, Ind PW, Northfield M, Shaw G. Added salmeterol versus higher-dose corticosteroids in asthma patients with symptoms on existing inhaled corticosteroid. Lancet 1994; 344: 219-224

${ }^{3}$ Woolcock A, Lundback B, Ringdal N, Jacques LA. Comparison of addition of salmeterol to inhaled steroids with doubling of the dose of inhaled steroids. Am J Respir Crit Care Med 1996; 153 : $1481-1488$

${ }^{4}$ Pauwels RA, Löfdahl CG, Postma DS, Tattersfield AE, O'Byrne P, Barnes PJ, Ullmann A. Effect of inhaled formoterol and budesonide on exacerbations of asthma. N Engl J Med 1997; 337: $1405-1411$

${ }^{5}$ Hancox RJ, Cowan JO, Flannery EM, Herbison GP, McLachlan CR, Wong CS, Taylor DR. Randomised trial of an inhaled $\beta_{2}$ agonist, inhaled corticosteroid and their combination in the treatment of asthma. Thorax 1999; 54: $482-487$

${ }^{6}$ Wiewroth R, Dauletbaev N, Buhl R. Wirksamkeit und Verträglichkeit einer Kombination von Fluticason und Salmeterol bei Patienten mit obstruktiver Atemwegserkrankung. Pneumologie 1997; $51: 14-20$

${ }^{7}$ Chung KF. The complementary role of glucocorticosteroids and long-acting $\beta$-adrenergic agonists. Allergy 1998; 53: 7-13

${ }^{8}$ Taylor DR, Hancox RJ. Interactions between corticosteroids and beta agonists. Thorax 2000; 55: 595-602

${ }^{9}$ Eickelberg O, Roth M, Lörx R, Bruce V, Rudiger J, Johnson M, Block LH. Ligand-independent activation of the gluccocorticoid receptor by $\beta_{2}$-adrenergic receptor agonists in primary human lung fibroblasts and vascular smooth muscle cells. J Biol Chem 1999; 274: 1005 - 1010

${ }^{10}$ Andersson F, Stahl E, Barnes PJ, Löfdahl CG, O’Byrne PM, Pauwels RA, Postma DS, Tattersfield AE, Ullmann A. Adding formoterol to budesonide in moderate asthmatics is cost-effective - results from the "FACET" study. Am J Respir Crit Care Med 2000; 161: A787 
11 Bisgaard $H$. Long-acting $\beta_{2}$-agonists in management of childhood asthma: a critical review of the literature. Pediatr Pulmonol 2000; 29: $221-234$

${ }^{12}$ Kips JC, O'Connor BJ, Inman MD, Svensson K, Pauwels RA, O'Byrne PM. A long-term study of the antiinflammatory effect of low-dose budesonide plus formoterol versus high-dose budesonide in asthma. Am J Respir Crit Care Med 2000; 161: 996-1001

${ }^{13}$ Chapman KR, Ringdal N, Backer V, Palmqvist M, Saarelainen S, Briggs M. Salmeterol and fluticasone propionated $(50 / 250 \mu \mathrm{g})$ administered via combination diskus inhaler: as effective as when given via separate diskus inhalers. Can Respir J 1999; 6: $45-51$

${ }^{14}$ Bateman ED, Britton M, Carrillo T, Almeida J, Wixon C. Salmeterol/ fluticasone combination inhaler. A new, effective and well tolerated treatment for asthma. Clin Drug Invest 1998; 16: 193 - 201

${ }^{15}$ Aubier M, Pieters WR, Schlosser NJ, Steinmetz KO. Salmeterol/ fluticasone propionate $(50 / 500 \mathrm{microg})$ in combination in a Diskus inhaler (Seretide) is effective and safe in the treatment of steroid-dependent asthma. Respir Med 1999; 93: 876-884

${ }^{16}$ Kavuru M, Melamed J, Gross G, Laforce C, House K, Prillaman B, Baitinger L, Woodring A, Shah T. Salmeterol and fluticasone propionate combined in a new powder inhalation device for the treatment of asthma: a randomized, double-blind, placebo-controlled trial. J Allergy Clin Immunol 2000; 105: 1108 - 1116

17 Jenkins C, Woolcock AJ, Saarelainen P, Lundback B, James MH. Salmeterol/fluticasone propionate combination therapy 50/ $250 \mu \mathrm{g}$ twice daily is more effective than budesonide $800 \mu \mathrm{g}$ twice daily in treating moderate to severe asthma. Respir Med 2000; 94: 715 - 723

18 Buhl R, Kardos P, Magnussen H, Matthys H, Sauer R, Schauer P, Vogelmeier C, Wettengel R, Worth H, Menz G. Feste Kombination inhalierbarer Kortikoide und langwirkender $\beta_{2}$-Sympathomimetika zur Langzeittherapie des Asthma bronchiale. Pneumologie 1999; 53: 210-212

${ }^{19}$ Zetterström O, Buhl R, Mellem H, Perpina M, Hedman J, O’Neill S. Budesonide and formoterol in a single inhaler, Symbicort ${ }^{\circledR}$, improves asthma control in adults: a randomised controlled study. Eur Respir J im Druck, 2001

${ }^{20}$ Zetterström O, Buhl R, Mellem H, Perpina M, Hedman J, O'Neill S, Peterson S. The new single inhaler product containing both budesonide/formoterol improves asthma control in adults. Eur Respir J 2000; 16: 455s

${ }^{21}$ Zetterström O, Buhl R, Mellem H, Perpina M, Hedman J, O’Neill S, Ekström T. Efficacy and safety of a new single inhaler product, containing both budesonide and formoterol in adult asthma. Eur Respir J 2000; 16: 455s

${ }^{22}$ Vermeulen JH, Simon G, Tal A, Vit P, Cobos N, Everara ML, De Boeck C, Peterson S. Improved lung function and rapid control achieved with the new single inhaler product containing both budesonide and formoterol in asthmatic children aged 4-17 years. Eur Respir J 2000; 16: 384s

${ }^{23}$ Tal A, Simon G, Vermeulen JH, Vit P, Cobos N, Everard ML, De Boeck $C$, Jerre $F$. The benefit of the new single inhaler product containing both budesonide and formoterol in asthmatic children. Eur Respir J 2000; 16: 384s

${ }^{24}$ Coutts JAP, Gibson NA, Paton JY. Measuring compliance with inhaled medication in asthma. Arch Dis Child 1991; 67: 332 - 333

${ }^{25}$ Balsbaugh TA, Chambers CV, Diamond JJ. Asthma controller medications: what do patients want? K Asthma 1999; 36: 591 596

${ }^{26}$ Kardos P, Brüggenjürgen B, Martin A, Meyer-Sabellek W, Richter K, Vogelmeier C, Willich SN, Buhl R. Die Behandlung des Asthma bronchiale nach einem bedarfsorientierten Behandlungsplan. Eingereicht 2001

${ }^{27}$ Cochrane GM. Compliance and outcomes in patients with asthma. Drugs 1996; 52: $12-19$
${ }^{28}$ Cramer JA, Mattson RH, Prevey MC, Scheyer RD, Ovellette VL. How often is medication taken as prescribed? JAMA 1989; 261: $3273-3277$

${ }^{29}$ Dompeling E, van Grunsven PM, van Schayck CP, Folgernig $\mathrm{H}$, Molema J, van Weel C. Treatment with inhaled steroids in asthma and chronic bronchitis: long term compliance and inhaler technique. Fam Pract 1992; 9: 161 - 166

${ }^{30}$ Gerrits CMJM, Herings RMC, Lammers JWJ, Leufkens HGM. Too low usage of inhaled steroids among patients with long-acting $\beta_{2}$-agonists. Eur Respir J 1998; 12: 156s-157s

${ }^{31}$ Horn CR, Essex E, Hill P, Cochrane GM. Does urinary salbutamol reflect compliance with the aerosol regimen in patients with asthma. Resp Med 1989; 83: 15-18

32 Bosley CM, Parry OT, Cochrane GM. Patient compliance with inhaled medication: does combining beta-agonists with corticosteroids improve compliance? Eur Respir J 1994; 7: 504-509

${ }^{33}$ Kelloway JS, Wyatt RA, Adlis SA. Comparison of patients' compliance with prescribed oral and inhaled asthma medications. Arch Int Med 1994; 154: 1349-1352

34 Barnes PJ, Woolcock AJ. Difficult asthma. Eur Respir J 1998; 12: 1209-1218

${ }^{35}$ Ankerst J, Persson G, Weibull E. A high dose of budesonid/ formoterol in a single inhaler was well tolerated by asthmatic patients. Eur Respir J 2000; 16: 33s

${ }^{36}$ British Thoracic Society. The british guidelines on asthma management. Asthma in adults and schoolchildren. Thorax 1995; 52: S2 - S8

${ }^{37}$ Wettengel R, Berdel D, Hofmann D, Krause J, Kroegel C, Kroidl RF, Leupold W, Lindemann H, Magnussen H, Meister A, Morr H, Nolte D, Rabe K, Reinhardt D, Sauer R, Schultze-Werninghaus G, Ukena D, Worth H. Asthmatherapie bei Kindern und Erwachsenen. Empfehlungen der Deutschen Atemwegsliga in der Deutschen Gesellschaft für Pneumologie. Med Klinik 1998; 93: 3950

${ }^{38}$ National Institutes of Health. Global strategy for asthma management and prevention NHLBI/WHO Workshop report. U.S. Department of Health and Human Services, Bethesda, 1993

${ }^{39}$ National Institutes of Health. International consensus report on diagnosis and management of asthma. U.S. Department of Health and Human Services, Bethesda, 1992

${ }^{40}$ Wallin A, Sandstrom T, Rosenhall L, Melander B. Time course and duration of bronchodilatation with formoterol dry powder in patients with stable asthma. Thorax 1993; 48: 611 -614

${ }^{41}$ Tattersfield AE, Löfdahl CG, Postma DS, Ekström T, Eivindson A, Schreurs A, Rasidakis A, Karlsson K, Larsson P. On demand treatment: comparision of formoterol and terbutaline in moderate asthma. Am J Respir Crit Care Med 1999; 159: A636

${ }^{42}$ Palmqvist M, Persson G, Lazer L, Rosenborg J, Larsson P, Lötvall J. Inhaled dry-powder formoterol and salmeterol in asthmatic patients: onset of action, duration of effect and potency. Eur Respir J 1997; 10: 2484-2489

${ }^{43}$ Dahl R. Comparative studies of inhaled salmeterol with other bronchodilators. Eur Respir J 1995; 5: 138 - 141

${ }^{44}$ Gillissen A, Buhl R, Kardos P, Magnussen H, Matthys H, Rabe KF, Rothe T, Russi EW, Schuaer J, Schmitz M, Vogelmeier C, Wettengel R, Worth H, Menz G. Inhalierbare Kortikosteroide in der Langzeittherapie der COPD. Stellungnahme eines Expertengremiums. Pneumologie 2000; 54: 256-262

Prof. Dr. A. Gillissen

Robert-Koch-Klinik

Nikolai-Rumjanzew-Str. 100

04207 Leipzig

E-mail: adrian.gillissen@sanktgeorg.de 